\title{
Angiogenesis in rat uterine scar after introduction after autological mesenchymal stem cells of bone marrow origin
}

\author{
Igor Maiborodin, Natalia Yakimova, Vera Matveeva, Andrey Shevela, Elena Maiborodina, \\ Ekaterina Pekareva, Olesya Tkachuk
}

Center of New Medical Technologies Institute of Chemical Biology and Fundamental Medicine, Novosibirsk, Russia. Email: $\underline{\text { imai@mail.ru }}$

Received 24 August 2010; revised 10 September 2011; accepted 13 September 2010.

\begin{abstract}
The results of injecting of autologic mesenchymal stem cells of bone marrow origin (AMSCBMO), transfected by the GFP gene, into the scar of rat uterine horns were studied by methods of light microscopy. After the introduction of AMSCBMO into the formed scar on the right ( 2 months after the ligation) large groups of blood vessels with cellular elements inside were present; groups like that were not found in the opposite side. Studying unstained sections under reflected ultraviolet light the sufficient bright luminescence in the endothelium and the external membrane of scar vessels was found in uterine horn only on the side of introduction of AMSCBMO. It was concluded that after the introduction of AMSCBMO into the scar tissue they form blood vessels by differentiation into endotheliocytes and pericytes. GFP gene expression not only in endothelium of vessels, but also in their external membrane indicates that differentiation of AMSCMBO is possible in endothelial and in pericytal directions.
\end{abstract}

Keywords: Uterine Scar; Mesenchymal Stem Cells; Angiogenesis; Endotheliocytes; Pericytes

\section{INTRODUCTION}

According to modern concepts, the physiological regeneration of tissues of the adult organism and their repair in case of damage occurs with direct participation of low-differentiated progenitor cells or stem cells. The main source of stem cells is bone marrow, capable, in addition to its basic - hematopoietic function, to generate precursors of cellular elements of a large number of body tissues.

Bone marrow contains two basic types of stem cells: hematopoietic and stromal (mesenchymal) [1]. It is believed that both of these types of cells are precursors with the potential to transdifferentiation into cells of different phenotypes [2].

In addition to bone marrow, pluripotent stem cells were also found in other tissues of the adult body - the adipose, muscle, brain, as well as in peripheral blood and blood from cord/placenta. Moreover, it is established that depending on a microenvironment the stem cells are able to get through haemopoetic/mesenchymal barrier as possess to high plasticity in case of a differentiation and a transdifferentiation.

J. M. Isner [3] isolated supposed endothelial progenitor cells from the bone marrow, some surface antigens of these cells were common to hematopoietic stem cells (CD34, CD133, Flk-1, Tie-2). These endothelial progenitor cells were injected systemically to immunodeficiency rats with pre-performed occlusion of coronary artery. Afterwards was discovered that donor cells were accumulated mainly in the areas of neoangiogenesis within the myocardium. Increasing the number of capillaries per unit volume associated with a significant improvement in function and parameters of the left ventricle compared with the control group. Similar work with similar results was performed on various models by other researchers [4-7].

H. Kamihata et al. [8] showed that intensive neoangiogenesis after implantation of bone marrow cells in the area of myocardial infarction was caused not only by direct participation of these cells, but also by significant expression of angiogenesis factors such as vascular endothelial growth factor, angiopoetin and others by these cells. M. Takahashi et al. [9] also supported the idea of paracrine mechanisms of the effect of bone marrow cells on the myocardium by inflammatory cytokines.

Since coronary heart disease is characterized by myocardial scarring, the expansion of the cavities and deterioration of the work of ventricles, which can be prevented only by full restoration of the myocardium, the studies were undertaken to assess the possibility of its restoration on the model of ischemic heart disease in 
mice. Cardiac progenitor cells were implanted in scar zone. Also local stimulation by hepatocyte growth factor and insulin-like growth factor-1 directly of the scar area was performed. These two factors have important characteristics, it is known that the hepatocyte factor is chemoattractant for cardiac progenitor cells. At the same time, insulin-like factor causes a strong cellular proliferation and increases the viability of these cells [10].

The results of this research showed decrease of the scar area by $42 \%$ with both methods of effects on the myocardium, as well as improved ventricular volume configuration. The reduction of scar area was associated with the formation of new myocardiocytes in which cardiac progenitor cells produce matrix metalloproteinases. In the analysis of chromosomes of myocardiocytes was shown that in the process of recovery of myocardium there is no "merger" of cells. As an alternative to introduction of stem cells the authors propose using a combination of growth factors [10].

The impoverishment of the population of circulating bone marrow cells with age - an important limiting condition for cellular therapy, as a preliminary preparation for the treatment in such cases it is suggested to use small molecules, polymers, growth factors or their combination [11].

In experiments on rats were showed that autologic mesenchymal stem cells of bone marrow origin (AMSCBMO) which hypoxically prepared within 24 hours before the transplantation into peri-infarction zone increase the expression of factors of vitality and vasculogenesis: hypoxia-inducible factor 1, angiopoietin-1, vascular endothelial growth factor, as well as receptors Flk-1, erythropoietin, Bcl-2 and Bcl-xL, that eventually leads to improved angiogenesis through increased paracrine mechanisms [12].

We can conclude that in the literature on treatment of scars with the use of cellular technologies, there are 2 main hypotheses about the destiny of implanted AMSCBMO. The first is that AMSCBMO in the continuation of their differentiation in hypoxic tissues are directly involved in angiogenesis and revascularization [4-7]. According to other researchers, the effect of AMSCBMO is mainly due to the expression of various factors of angiogenesis and other cytokines $[8,9,12]$. M. Rota et al. [10] believe that there is a combination of direct involvement of stem cells with paracrine mechanisms. So, there is no common opinion and until there is remained not clear what happens to AMSCBMO after implantation into the tissues and organs of the body.

\section{AIM}

These days inflammatory diseases of female genitals and, as a result of them, adhesions, lead to reproductive dis- orders in women. The most relevant in this regard is infertility. Studying peculiarities of etiopathogenesis in the development of adhesions and synechiae in the uterine cavity allows justifying the search of new directions of their treatment and prevention.

Due to the low efficiency of widely used techniques for treating scars of myometrium the attempt to correct the pathology with the use of cellular technologies was made. In addition, currently remains unexplored the destiny of stem cells after the introduction one way or another into the organism.

An attempt to use autologic mesenchymal stem cells of bone marrow origin (AMSCBMO) to accelerate the regeneration of scar of myometrium in the experiment was made due to the numerous reports on the effectiveness of cellular technologies in the treatment of coronary heart disease [3-10,12]. Cellular-mediated strategies in the treatment of this pathology are based on the implantation directly into the ischemic myocardium or the coronary vessels of bone marrow cells. This serves two purposes: myocardial revascularization and eliminating the deficit of functional cellular elements in myocardium.

\section{MATERIAL AND METHODS}

In our research the female Wag-rats weighing 180-200 g at the age of 6 months were used as models. In aseptic conditions lower midline laparotomy was performed. Uterine horns were taken out into the wound and carefully rounded by sterile gauze. At the end of each horn near the corpus uteri were placed ligatures from catgut and bandaged up in that part. Abdominal cavity was sutured tightly layer-by-layer.

Isolation of mesenchymal stem cells: AMSCBMO isolated by washing out the bone marrow from femur epiphysis that taken under ether anesthesia in male rats, inbreeding line Wag. Obtained suspension of cells was placed in plastic bottles ("Nunk", Denmark), in 48 hours after explantation of bone marrow the cells that did not attach were removed. Attached cells were cultured in $\alpha$-MEM medium with supplement of $10 \%$ fetal calf serum ("Biolot", Russia) at $37^{\circ} \mathrm{C}$ in $\mathrm{CO}_{2}$-incubator with $5 \% \mathrm{CO}_{2}$ in saturated humidity. The culture medium was changed every three days. Performing subculturing the monolayer culture was dispersed with density of 10005000 cells $/ \mathrm{sm}^{2}$ (depending on the growth properties of used fetal serum), were used standard solutions of Versene and tripsin.

Transfection: Mesenchymal AMSCBMO from second passage, obtained from rats of mentioned line, transfected DNA of plasmid pEGFP-N1 (Clontech Laboratories), which contained the gene of green fluorescent protein (GFP) under the control of cytomegalovirus promoter and neomycin resistance gene under the control of 
the promoter of the virus SV40, that necessary for the subsequent selection using geneticyn G418 (pEGFP-N1; Clontech Laboratories). Transfection was performed in the presence of the reagent for transfection TurboFect (Fermentas) as recommended by the manufacturer; the protocol for transfection of cellular suspension was used. Transfection was performed using $1 \times 10^{6}$ cells in $1 \mathrm{ml}$ of the suspension, $4 \mathrm{mg}$ plasmid DNA and $10 \mathrm{ml}$ of reagent for transfection (TurboFect). Cells after transfection were cultured in $\alpha$-MEM medium with supplement of $10 \%$ fetal calf serum (Biolot, Russia), using a selection of G-418 (Sigma) at a concentration of $400 \mathrm{ng} / \mathrm{ml}$.

As specific unique markers for AMSCBMO are unknown, for identification of these cells were used physical, morphological and functional properties. Untransfected and transfected cells (for demonstration that of AMSCBMO properties after transfection remain without changes) were characterized by the following physical, morphological and functional properties which are peculiar for mesenchymal stem cells cultured in vitro: attachment to the surface of the cultivation, morphology, proliferation, formation of colonies of fibroblast-like cells and the ability to induced differentiation in bone tissue.

Using methods of light and fluorescent microscopy and cytological staining methods was showed that the cultured untransfected and transfected rat bone marrow cells in vitro: were attached to the surface of cultivation, had fibroblast-like morphology continued at all times of cultivation, maintained in culture for several subcultures, formed colonies of fibroblast-like cells when were dispersed at low density, in the presence of a linear-specific factors differentiated into cells of the bone tissue. For research the untransfected cells of $0-3$ passages and transfected cells of 0-2 passage were used.

However, the physical, morphological and phenotypical characteristics are not unique criteria that can be used for specific identification of AMSCBMO. AMSCBMO ability for induced differentiation in vitro into bone, adipose tissue and cartilage is the only critical requirement for determining of prospective populations of stem cells.

Induction of osteogenic differentiation of mesenchymal stem cells in vitro: For induction of osteogenic differentiation the $0.1 \mu \mathrm{M}$ desoximethasone, $50 \mu \mathrm{M}$ ascorbic acid and $10 \mathrm{mM} \beta$-glycerophosphate (Sigma) was used. As AMSCBMO have properties to differentiation into cells of bone tissue, the conditions of its implementation are most reproducible. Such differentiation is typically used to characterize the cultures of stem cells in vitro and is typical way by default for the majority of AMSCBMO in culture.

Osteogenic differentiation was determined by two markers: the alkaline phosphatase activity and mineralization of extracellular matrix by calcium ions: Cyto- chemical detection of alkaline phosphatase was performed by using nitrotetrazolium blue in the presence of substrate for alkaline phosphatase of 5-bromo-4-chloro3-indolyl. Accumulation of calcium in the extracellular matrix was recorded by alizarin red staining.

In 4 hours after the transfection the cells were diluted with untransfected cells at a ratio of $1: 2.5$, respectively, and $0.1 \mathrm{ml}$ of the mixture was injected during procedure of relaparotomy and after removal of residual unlysed suture material into the region of formed scar within 2 months after ligation of right uterine horns of female rats Wag.

Remaining cells after transplantation cultured for 10 days to evaluate the efficiency of transfection and stability of gene expression. Expression of introduced gene GFP by rat mesenchymal AMSCBMO was assessed visually under a fluorescent microscope, directly looking at culture in 48 hours after the transfection. The efficiency of transfection was assessed as a percentage of luminescent cells relative to all cells. The percentage of transfected cells in the diluted culture was about 3\%.

As in most cases, using technology based on the introduction of plasmid DNA was observed only a temporary expression of the introduced GFP gene in rat AMSCBMO. Cultivation of cells, transfected by plasmid pEGFP-N1, without selection (not using geneticyn (G418, Sigma)) showed a decrease in the number of cells synthesizing a green fluorescent protein, as a result of their replacement by untransfected cells. Nevertheless, after 1 week in culture of transfected cells of first passage, seeded with density of 5000 cells per $1 \mathrm{~cm} 2$, were observed cells that synthesized green fluorescent protein. Thus, as in most cases, using technology based on the introduction of plasmid DNA with cationic lipids, without subsequent selection for creation of stable clone, was obtained culture of rat AMSCBMO temporarily expressing green fluorescent protein.

Fragments of the uterine horns with scar tissues and synechiae, biopsied after $1,2,3$ and 4 weeks after the introduction of AMSCBMO, were preserved in a $4 \%$ paraformaldehyde on biphosphate buffer $(\mathrm{pH}$ 7.4) for at least 24 hour, dehydrated in a gradien of ethanol, lightened in xylene and embedded in paraffin. Unpainted microscopic sections 5-7 microns thick were studied under a light microscope Axioimager M1 (Carl Zeiss, Germany) with a magnification of up to 1500 times in the luminescence mode with a filter Alexa 488. The scar of left uterine horn in a corresponding time after the introduction of a suspension AMSCBMO and myometrium scar of animals 2 months after ligation of uterine horns with no subsequent use AMSCBMO were used as the controls. 6 animals were examined at each point of the experiment. 
All studies were performed in compliance with "Rules for work using experimental animals”, all manipulations were performed under general anesthesia by ether inhalation.

\section{RESULTS}

After 2 months there was detected a moderate development of adhesions in the pelvic area without involvement of the upper part of abdomen. There were discovered expressed changes in the uterine horns, which were manifested by presence of hydrometra above the place of ligation to the isthmic part of uterine tube, probably due to lack of outflow into tube and further into the abdominal cavity. The content in the uterine horn was transparent, serous, without the presence of purulent elements. At this time in place of ligation of rat uterine horns the encapsulated suture materials were present, despite the data on the timing of resorption of catgut in 15-20 days in humans. It should be noted that we have already drawn attention in previous publications at later dates of resorption of "lyzed" suture material [13].

In the time of study of the uterine horn structure, using magnification in 6-10 times, the formation of synechiae in the site of ligation, hypertrophy and stretching of the uterine wall, formation of complete obstruction were discovered. In the area of narrowing of the uterine horns the atrophy of the endometrium and myometrium was found.

In 1 week after the introduction of AMSCBMO into formed scar on the right side there were large groups of blood vessels with cellular elements inside (Figure 1), the such groups of vessels were not found in the scar on the left side.

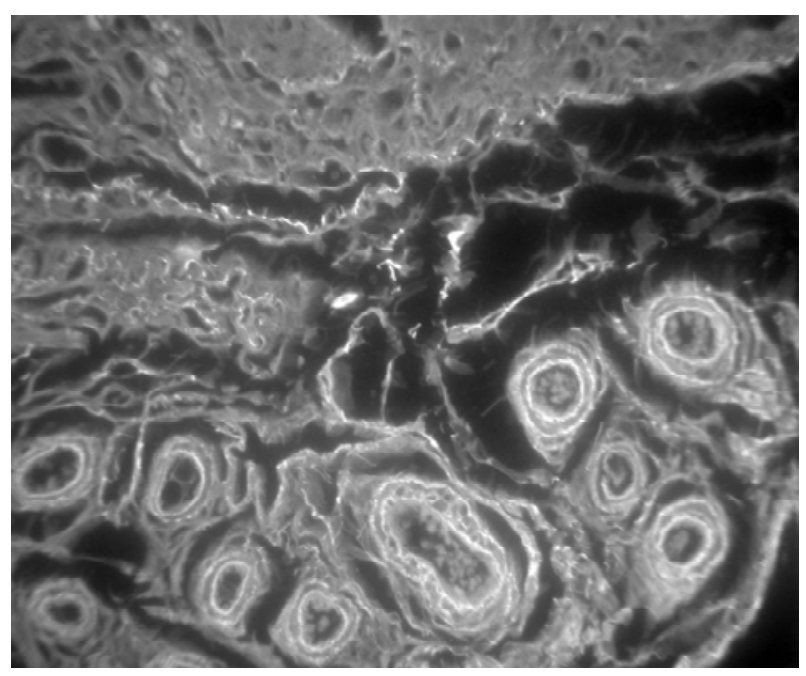

Figure 1. Groups of blood vessels in a scar of right rat uterine horn in 1 week after application of AMSCBMO, in the many vessels the luminescence of accurately outlined endothelium and an external membrane is present. Magnification $240 \times$.
In the study of unstained sections of uterine scar in the reflected ultraviolet light sufficiently the bright luminescence in the endothelium and the external membrane of scar vessels of the right uterine horn was found (Figure 1). Also in the endometrium and myometrium the slight edema and many small luminous objects, most likely, vessels of capillary type were found (Figure 2).

In 2 weeks after the introduction of AMSCBMO the very bright luminescence in the endothelium and adventitia of large vessels in scar of the right uterine horn was discovered. The endothelium and the external membrane of such vessels were represented in the form of bright, clear, well defined lines (Figure 3). In the myometrium

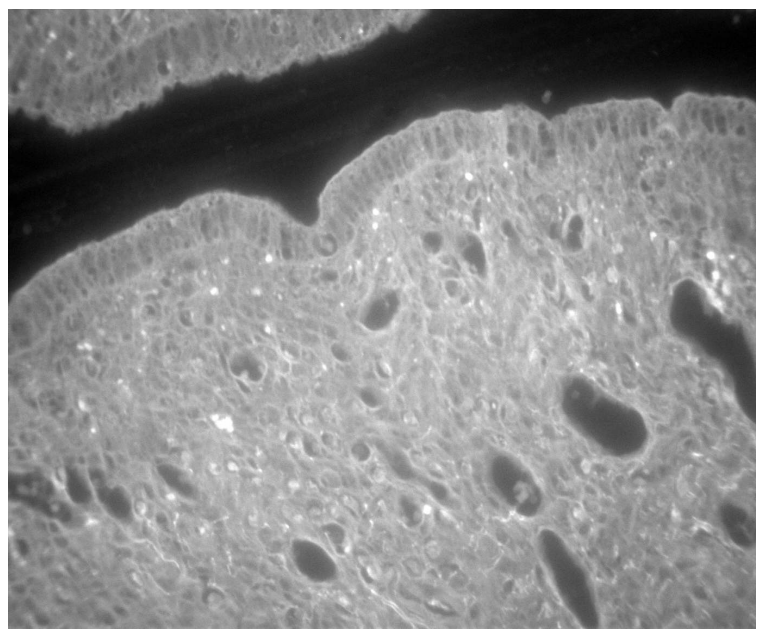

Figure 2. In 1 week after introduction of AMSCBMO in endometrium and myometrium of the right uterine horn the set of small objects with luminescence (vessels of capillary type) and the signs of interstitial edema are present. Magnification $240 \times$.

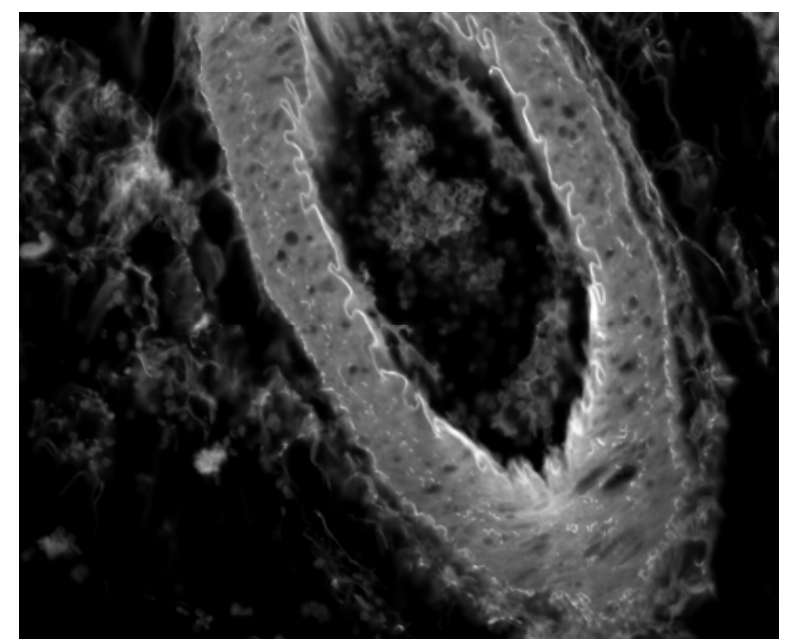

Figure 3. In 2 weeks after injection of AMSCBMO the enough bright luminescence is revealed in endothelium and adventitia of large vessels in scar of the right uterine horn. Vessel membranes with luminescence are presented by accurate, well limited lines. Magnification $630 \times$. 
and endometrium of the left uterine horn at this time the luminescent structures were completely absent (Figure 4).

After 3 weeks only in the wall of some blood vessels on the right was noted weak luminescence, almost at the level of background (Figure 5).

By week 4 the luminescence intensity of the vascular walls and the number of vessels in the structures, in which was marked luminescence, was even more reduced (Figure 6).

On the opposite side for all periods of study the luminescence of the vascular walls were at the level of background, is necessary to repeat that large groups of vessels were absent. In the myometrium and endometrium small luminous objects were absent, but at the same time

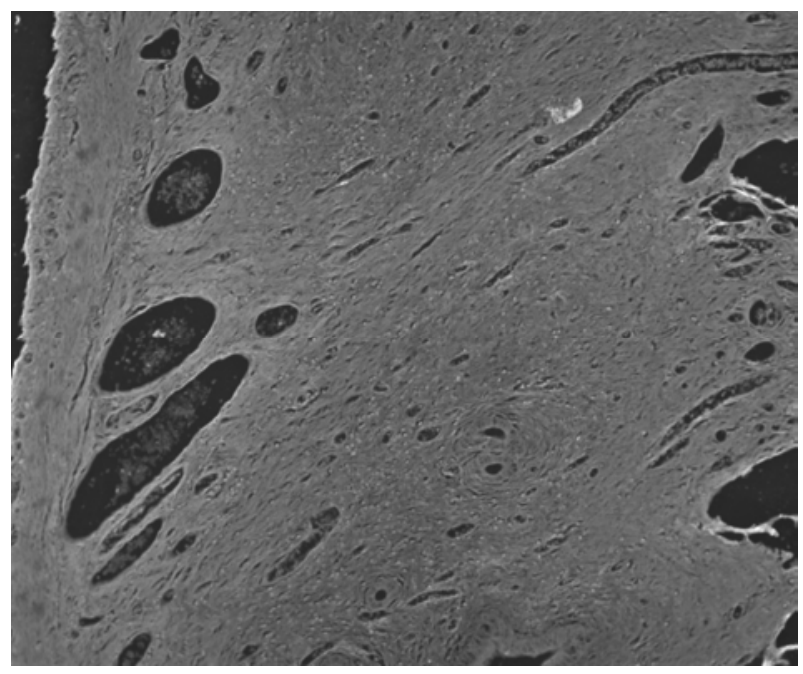

Figure 4. Absence of structures with luminescence and signs of edema in endometrium and myometrium of right uterine horn in 2 weeks after use of AMSCBMO. Magnification $240 \times$.

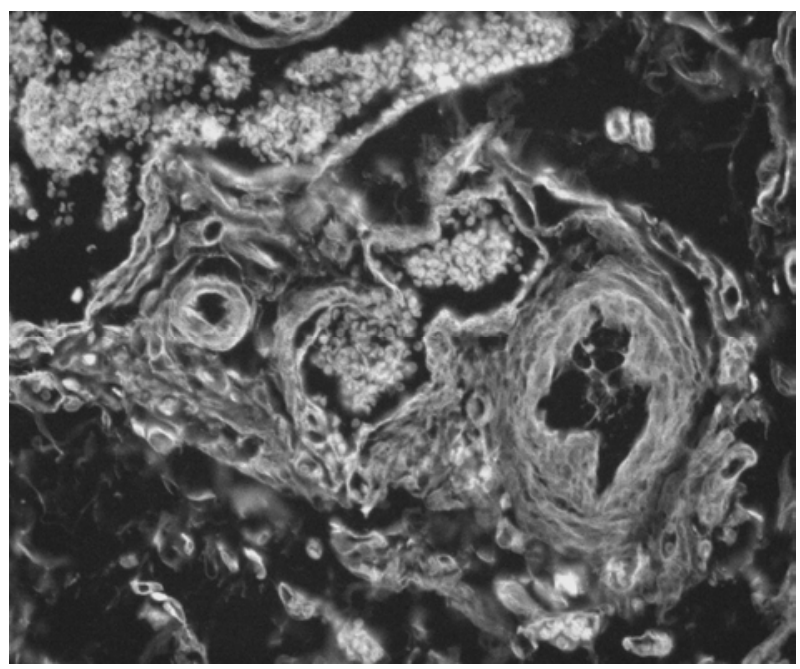

Figure 5. The weak luminescence, practically at background level, is present in a wall of some blood vessels in 3 weeks after introduction of AMSCBMO. Magnification $240 \times$. there were no signs of edema (Figures 7-11). In the scar of the uterine horn of animals without the AMSCBMO introduction (control) the luminescence objects were absent in vessels, in the structure of the scar and in surrounding tissues (Figure 12).

Unfortunately, after using AMSCBMO the more rapid resorption of the scar and reduction of hydrometra were not found. But, this preliminary study was conducted on a small number of observations. For accurate conclusions about the effectiveness of this procedure regarding the status of the scar there is necessary to repeat this experiment with larger number of animals and to ob serve longer periods after the introduction of AMSCBMO.

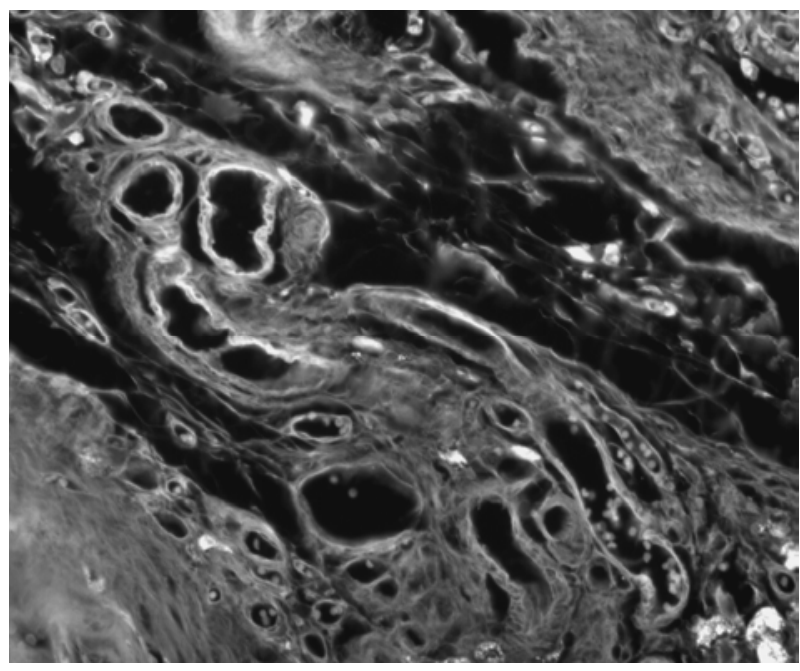

Figure 6. In 4 weeks after application of AMSCBMO the weak luminescence remains in a wall of only individual vessels. Magnification $240 \times$.

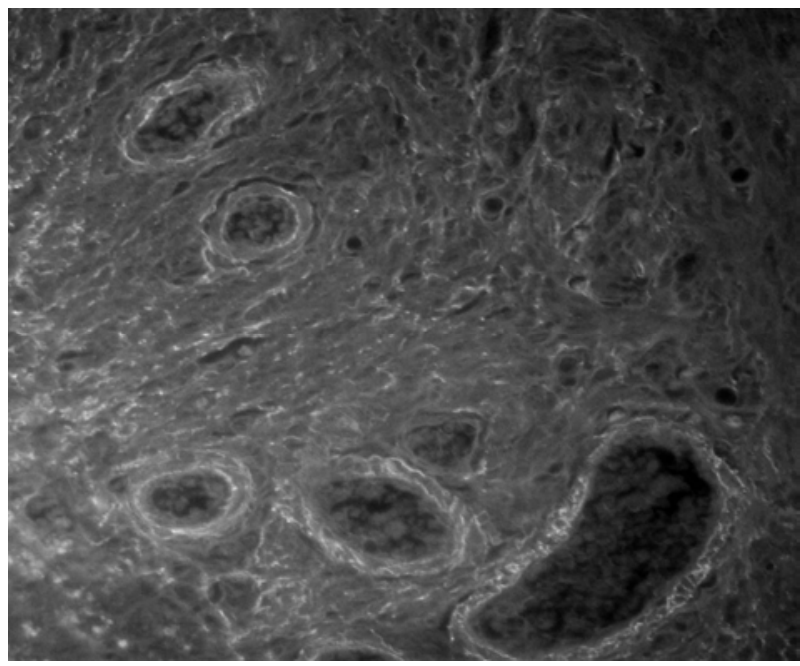

Figure 7. In 1 week after introduction of AMSCBMO in a scar of rat left uterine horn the number of vessels is much less, a luminescence of their structures is practically at background level. Magnification $320 \times$. 


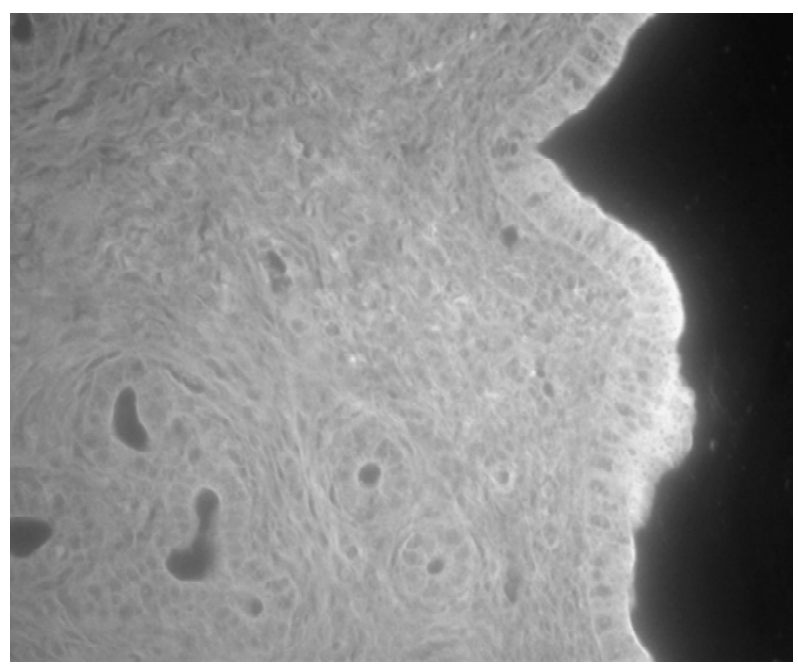

Figure 8. In left uterine horn the objects with luminescence and signs of edema in 1 week after injection of AMSCBMO are absent. Magnification $240 \times$.

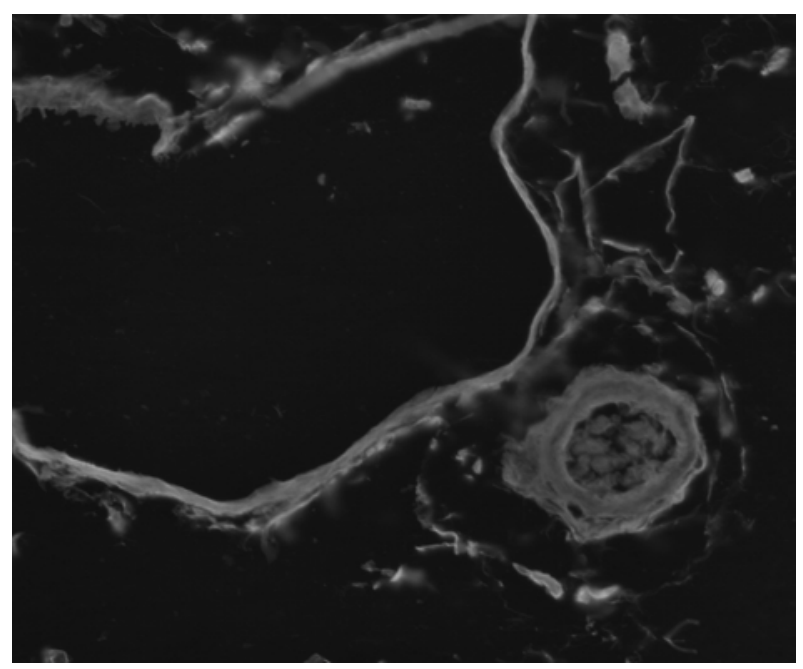

Figure 9. The absence of signs of edema and structures with luminescence in vessels of a scar of left uterine horn in 2 weeks after use of AMSCBMO. Magnification $240 \times$.

\section{DISCUSSIONS}

Rat uterine horn is a thin tube, one end of which connects with the uterine cavity, the other - with the fallopian tube, opening into the peritoneal cavity near the ovarian surface. Normally in mammals there is outflow of content from fallopian tubes into the peritoneal cavity with subsequent resorption by peritoneum. After ligation of uterine horns in this section takes place ischemia followed by necrosis, scar and hydrometra formation. These changes are clearly seen in macropreparation with a slight magnification.

Concerning the results of use of AMSCBMO it is necessary to pay attention to the development of the vascular net at the injection site (Figures 1 and 3-5). The proof, that these groups of vessels were formed precisely as a result of AMSCBMO and from them, is the luminescence of walls of these vessels (Figures 1 and 3-5). GFP gene, introduced in the DNA of AMSCBMO, transfers without changes to daughter cells and cells of next generations. These cells and structures formed from them are also shone in the reflected ultraviolet light.

Until recently by prevailing dogma was that the angiogenic response, which develops in postnatal life, occurs because of the growth of existing capillaries. However, now it is convincingly proven that a small, but biologically significant portion of endothelial cells involved in the formation of new capillaries, have bone marrow origin [14-16].

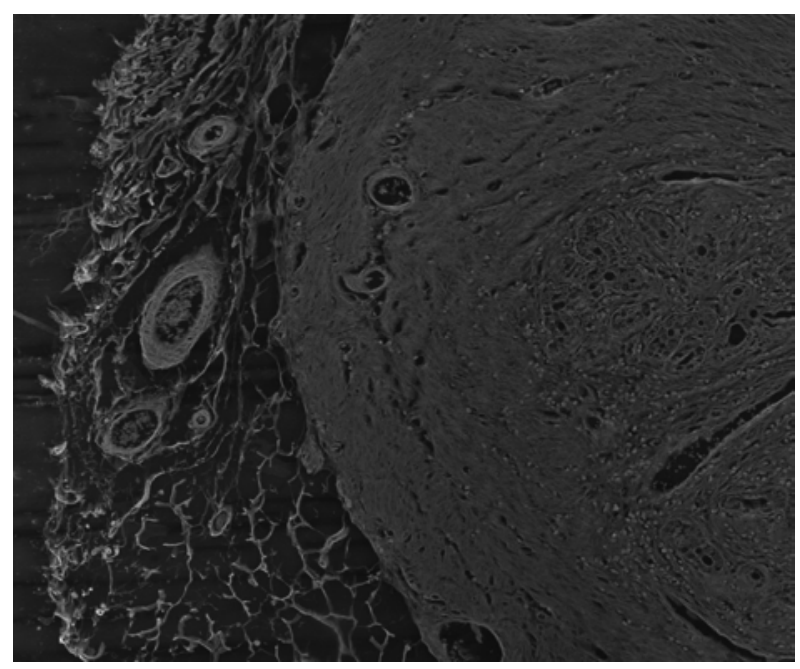

Figure 10. In 3 weeks after injection of AMSCBMO the objects with luminescence are absent in structures of left uterine horn and surrounding tissues. Magnification $180 \times$.

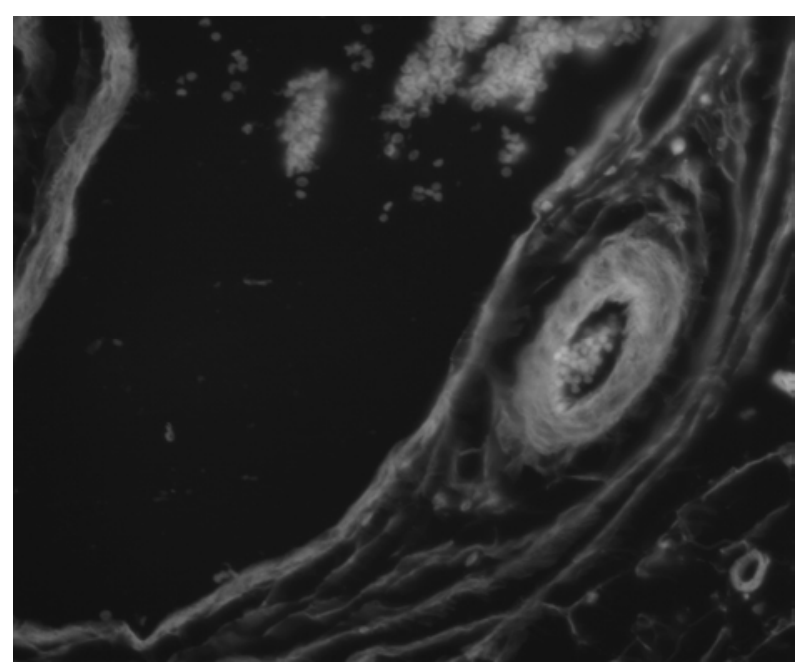

Figure 11. Absence of a luminescence in vessels of scar of left uterine horn in 4 weeks after introduction of AMSCBMO. Magnification $480 \times$. 


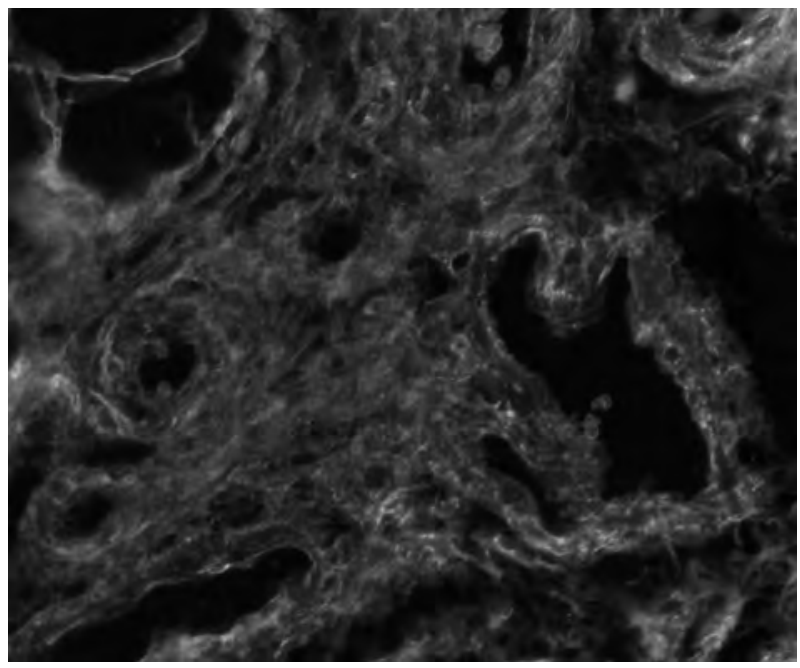

Figure 12. A control animal without introduction of AMSCBMO. The objects with luminescence in scar structures of uterine horn completely are absent. Magnification $480 \times$.

Endothelial cells divide every three years, but in the case of retinal vessels - every 14 years. However endotheliocytes can be induced to replication as response to physiological or pathological stimulation. In some cases, bone marrow populations of endothelial progenitor cells can be completely replaced every five days. This is a real advantage when the physiological circumstances require increased blood supply, both in healing wounds, and in preparation of a fertilized egg for the implantation in the richly vascularized endometrium [17].

In 1962 was first demonstrated the possibility of existence of circulating endothelial cells. It was shown that on Dacron patch, which was isolated from contact with the surrounding tissues and was located in the wall of the current vascular prosthesis of the aorta (experimental prosthetics of the pig thoracic aorta), connective tissue is forming. After 14 days and later after implantation of Dacron, there was determined a layer of endothelial and other cells, including mononuclear cells, fibroblasts, as well as giant cells of foreign bodies. Detected cells could have only one source-the circulating blood $[18,19]$.

In experiments on dogs was shown that in a few days on the surfaces of endothelial impermeable vascular prostheses the isolated islands of endothelial cells are appeared, as when Dacron grafts was installed in inferior vena cava and as in thoracic aorta. This also confirms that the source of endothelial cells - circulating blood [15]. These results were confirmed when after total irradiation of dogs the bone marrow transplantation from animals of another kind was made, in which the donor DNA of circulating endothelial cells were clearly different from the DNA of recipient cells [16].

Blood vessels are forming by two interacting cellular types. They are endothelial cells lining the inner surface of the bloodstream and perivascular cells, named pericytes, enveloping the outer surface of the vascular tubes [20]. Pericytes are not only included in the hemodynamic process, but also play an active role in the formation of blood vessels. Some researchers hypothesize that endothelial cells and pericytes have common precursor with hematopoietic cells. This hypothesis is based on the fact that developing hematopoietic and endothelial cells have common surface markers and that hematopoietic cells can be developed from embryonic cells of major blood vessels [21-23].

Most commonly origin of pericytes associate with mesenchymal stem cell [24]. After the initiation of differentiation the progenitor of pericytes chemotactically adhere to endothelial cells in the capillary plexus in the time of beginning of angiogenesis [25]. In addition, there are reports that pericytes can be generated from endothelial cells during their transdifferentiation. This was discovered in the posterior aorta [26], as well as in heart valves [27].

The luminescence of the endothelium and the external membrane of the vessels, discovered in our studies (Figures 1 and 3-5), indicates that AMSCBMO directly rather than indirectly (through cytokines or other cellular signals), were involved in the formation of blood vessels, possibly because of the presence of pluripotent cells which already were stimulated to differentiate into endothelial or pericytal directions. It is also possible that tissue hypoxia, as a result of ligation of structures together with the vessels, stimulates the differentiation of introduced AMSCBMO into endotheliocytes [12].

Additional evidence of angiogenesis, as a result of application of AMSCBMO, is the groups of vessels in the scar that were found only on the right - the side of AMSCBMO introduction (Figures 1-6), but not in the other side (Figures 7-11) and not in the control (Figure 12).

It is necessary to attract attention to another aspect of the findings. Expression of introduced GFP gene not only in the endothelium of blood vessels, but also in the external membrane (Figures $\mathbf{1}$ and 3), is most likely a sign that endotheliocytes and pericytes have the same progenitor cells, or that differentiation of AMSCBMO is possible as well as in endothelial and in pericytal directions. We have already pointed out that, in the opinion of many researchers, endothelial cells and pericytes have common precursor with hematopoietic cells [21-23].

Besides that, the unilateral development of blood vessels and the presence of luminescence objects in walls (Figures 1 and 3-5) indicate that in this case introduced AMSCBMO do not migrate from the site of injection, do not destroy, and they are not only "building material" or 
"signal" to the beginning of angiogenesis for own cells of the donor organism . In all these cases, of course, it is possible that luminescence protein and transfected GFP gene leave the destroyed AMSCBMO and then are absorbed by neighbor cells. However, proteins and DNA fragments that got into cells by way of phagocytosis or pinocytosis, degraded with the loss of ability to luminescence and insertion into the genome. So, when AMSCBMO are destroyed, intensity of luminescence should be minimal (the protein GFP leaves AMSCBMO) and very quickly should entirely disappear; more so in this case should not be clearly defined and limited luminous structures.

The decrease of light intensity in the vessels of the scar in the uterine horn after introduction of AMSCBMO with time, probably due to the gradual restoration of genome of transfected cells or the displacement of AMSCBMO by own cells.

Attention should be paid, that the reduction of number of objects, which can synthesize green fluorescent protein, was showed during cultivation of cells, transfected by plasmid pEGFP-N1 without selection, as a result of their replacement by untransfected cells.

In conclusion, it should be noted that after increasing the number of vessels, especially "young" with thin walls, metabolic processes in tissues with scar improve. As a result of optimizing the conditions of life and functioning of fibroblasts the exchange of components of extracellular matrix of scar connective tissue may intensify, rejuvenation of collagen and elastin fibers may occur, that will further manifest by appearance of thinner structures, ordering of their location [28] and passableness of uterine horns with formed adhesions can be restored.

\section{CONCLUSIONS}

1) After introduction into uterine scar of AMSCBMO the increase of number of vessels due to the processes of neoangiogenesis was detected. In this case the AMSCBMO do not migrate and were not destroyed in the site of introduction, but form the blood vessels by differentiation into endotheliocytes and pericytes. In 1 week in rats after introduction of AMSCBMO the vessels formed from these cells are already fully functional, consist of all the structural walls and contain blood cells.

2) Expression of GFP gene not only in the endothelium of blood vessels, but also in their outer membranes indicates that differentiation of AMSCBMO in endothelial and in pericytal directions is possible.

\section{REFERENCES}

[1] Campagnoli, C., Roberts, I.A., Kumar, S., Bennett, P.R.,
Bellantuono, I. and Fisk, N.M. (2001) Identification of mesenchymal stem/progenitor cells in human first-trimester fetal blood, liver, and bone marrow. Blood, 98, 2396-2402. doi:10.1182/blood.V98.8.2396

[2] Huss, R. (2000) Isolation of primary and immortalized CD34-hematopoietic and mesenchymal stem cells from various sources. Stem Cells, 18, 1-9.

doi:10.1634/stemcells.18-1-1

[3] Isner, J.M. (2000) Tissue responses to ischemia: Local and remote responses for preserving perfusion of ischemic muscle. The Journal of Clinical Investigation, 106, 615-619. doi:10.1172/JCI10961

[4] Fukushima, S., Varela-Carver, A., Coppen, S.R., Yamahara, K., Felkin, L.E., Lee, J., Barton, P.J., Terracciano, C.M., Yacoub, M.H. and Suzuki, K. (2007) Direct intramyocardial but not intracoronary injection of bone marrow cells induces ventricular arrhythmias in a rat chronic ischemic heart failure model. Circulation, 115, 2254-2261. doi:10.1161/CIRCULATIONAHA.106.662577

[5] Grauss, R.W., Winter, E.M., van Tuyn, J., Pijnappels, D.A., Steijn, R.V., Hogers, B., van der Geest, R.J., de Vries, A.A., Steendijk, P., van der Laarse, A., Gittenberger de Groot, A.C., Schalij, M.J. and Atsma, D.E. (2007) Mesenchymal stem cells from ischemic heart disease patients improve left ventricular function after acute myocardial infarction. American Journal of Physiology. Heart and Circulatory Physiology, 293, H2438-H2447. doi:10.1152/ajpheart.00365.2007

[6] Jackson, K.A., Majka, S.M., Wang, H., Pocius, J., Hartley, C.J., Majesky, M.W., Entman, M.L., Michael, L.H., Hirschi, K.K. and Goodell, M.A. (2001) Regeneration of ischemic cardiac muscle and vascular endothelium by adult stem cells. The Journal of Clinical Investigation, 107, 1395-1402. doi:10.1172/JCI12150

[7] Kocher, A.A., Schuster, M.D., Szabolcs, M.J., Takuma, S., Burkhoff, D., Wang, J., Homma, S., Edwards, N.M. and Itescu, S. (2001) Neovascularization of ischemic myocardium by human bone-marrow-derived angioblasts prevents cardiomyocyte apoptosis, reduces remodeling and improves cardiac function. Nature Medicine, 7, 430-436. doi:10.1038/86498

[8] Kamihata, H., Matsubara, H., Nishiue, T., Fujiyama, S., Tsutsumi, Y., Ozono, R., Masaki, H., Mori, Y., Iba, O., Tateishi, E., Kosaki, A., Shintani, S., Murohara, T., Imaizumi, T. and Iwasaka, T. (2001) Implantation of bone marrow mononuclear cells into ischemic myocardium enhances collateral perfusion and regional function via side supply of angioblasts, angiogenic ligands, and cytokines. Circulation, 104, 1046-1052.

doi:10.1161/hc3501.093817

[9] Takahashi, M., Li, T.S., Suzuki, R., Kobayashi, T., Ito, H., Ikeda, Y., Matsuzaki, M. and Hamano, K. (2006) Cytokines produced by bone marrow cells can contribute to functional improvement of the infarcted heart by protecting cardiomyocytes from ischemic injury. American Journal of Physiology. Heart and Circulatory Physiology, 291, H886-H893. doi:10.1152/ajpheart.00142.2006

[10] Rota, M., Padin-Iruegas, M.E., Misao, Y., de Angelis, A., Maestroni, S., Ferreira-Martins, J., Fiumana, E., Rastaldo, R., Arcarese, M.L., Mitchell, T.S., Boni, A., Bolli, R., Urbanek, K., Hosoda, T., Anversa, P., Leri, A. and Kajstura, J. (2008) Local activation or implantation of 
cardiac progenitor cells rescues scarred infarcted myocardium improving cardiac function. Circulation Research, 103, 107-116. doi:10.1161/CIRCRESAHA.108.178525

[11] Dimmelerm, S. and Leri, A. (2008) Aging and disease as modifiers of efficacy of cell therapy. Circulation Research, 102, 1319-1330. doi:10.1161/CIRCRESAHA.108.175943

[12] Hu, X., Yu, S.P., Fraser, J.L., Lu, Z., Ogle, M.E., Wang, J.A. and Wei, L. (2008) Transplantation of hypoxia-preconditioned mesenchymal stem cells improves infarcted heart function via enhanced survival of implanted cells and angiogenesis. The Journal of Thoracic and Cardiovascular Surgery, 135, 799-808. doi:10.1016/j.jtcvs.2007.07.071

[13] Maiborodin, I.V., Maiborodina, E.I., Iakimova, N.V., Motorina, I.P. and Pekarev, O.G. (2008) Absorbable suture material in the body. Arkhiv Patologii, 70, 51-53.

[14] Carmeliet, P. and Luttun, A. (2001) The emerging role of the bone marrow-derived stem cells in (therapeutic) angiogenesis. Thrombosis and Haemostasis, 86, 289-297.

[15] Shi, Q., Wu, M.H., Hayashida, N., Wechezak, A.R., Clowes, A.W. and Sauvage, L.R. (1994) Proof of fallout endothelialization of impervious dacron grafts in the aorta and inferior vena cava of the dog. Journal of Vascular Surgery, 20, 546-557.

[16] Shi, Q., Rafii, S., Wu, M.H., Wijelath, E.S., Yu, C., Ishida, A., Fujita, Y., Kothari, S., Mohle, R., Sauvage, L.R., Moore, M.A., Storb, R.F. and Hammond, W.P. (1998) Evidence for circulating bone marrow-derived endothelial cells. Blood, 92, 362-367.

[17] Carmeliet, P. and Jain, R.K. (2000) Angiogenesis in cancer and other diseases. Nature, 407, 249-257. doi:10.1038/35025220

[18] Poole, J.C., Sabiston Jr, D.C., Florey, H.W. and Allison, P.R. (1962) Growth of endothelium in arterial prosthetic grafts and following endarterectomy. Surgical Forum, 13, 225-227.

[19] Stump, M.M., Jordan Jr, G.L., Debakey M.E. and Halpert, B. (1963) Endothelium grown from circulating blood on isolated intravascular dacron hub. The American Journal of Pathology, 43, 361-367.

[20] Bergers, G. and Song, S. (2005) The role of pericytes in blood-vessel formation and maintenance. Neuro-Oncology, 7, 452-464. doi:10.1215/S1152851705000232

[21] Carmeliet, P. (2004) Manipulating angiogenesis in medicine. Journal of Internal Medicine, 255, 538-561. doi:10.1111/j.1365-2796.2003.01297.x

[22] Cho, H., Kozasa, T., Bondjers, C., Betsholtz, C. and Kehrl, J.H. (2003) Pericyte-specific expression of rgs5: implications for PDGF and EDG receptor signaling during vascular maturation. The FASEB Journal, 17, 440-442.

[23] Ribatti, D., Vacca, A., Nico, B., Ria, R. and Dammacco, F. (2002) Cross-talk between hematopoiesis and angiogenesis signaling pathways. Current Molecular Medicine, 2, 537-543. doi:10.2174/1566524023362195

[24] Creazzo, T.L., Godt, R.E., Leatherbury, L., Conway, S.J. and Kirby, M.L. (1998) Role of cardiac neural crest cells in cardiovascular development. Annual Review of Physiology, 60, 267-286. doi:10.1146/annurev.physiol.60.1.267

[25] Hellström, M., Kalén, M., Lindahl, P., Abramsson, A. and Betsholtz, C. (1999) Role of PDGF-B and PDGFR-beta in recruitment of vascular smooth muscle cells and pericytes during embryonic blood vessel formation in the mouse. Development, 126, 3047- 3055.

[26] Gittenberger-de Groot, A.C., DeRuiter, M.C., Bergwerff, M. and Poelmann, R.E. (1999) Smooth muscle cell origin and its relation to heterogeneity in development and disease. Arteriosclerosis, Thrombosis, and Vascular Biology, 19, 1589-1594.

[27] Nakajima, Y., Mironov, V., Yamagishi, T., Nakamura, H. and Markwald, R.R. (1997) Expression of smooth muscle alpha-actin in mesenchymal cells during formation of avian endocardial cushion tissue: a role for transforming growth factor beta3. Developmental Dynamics, 209, 296-309. doi:10.1002/(SICI)1097-0177(199707)209:3<296::AIDAJA5>3.0.CO;2-D

[28] Tsuji, T. and Sawabe, M. (1988) Elastic fibers in striae distensae. Journal of Cutaneous Pathology, 15, 215-222. doi:10.1111/j.1600-0560.1988.tb00547.x 